\title{
Preparatory procedure and equipment for the European x-ray free electron laser cavity implementation
}

\author{
D. Reschke, ${ }^{*}$ R. Bandelmann, T. Buettner, K. Escherich, A. Goessel, B. v.d.Horst, J. Iversen, D. Klinke, G. Kreps, \\ N. Krupka, L. Lilje, A. Matheisen, W.-D. Moeller, H. Morales Zimmermann, C. Mueller, B. Petersen, D. Proch, \\ M. Schmoekel, N. Steinhau-Kuehl, J.-H. Thie, H. Weise, and H. Weitkaemper \\ DESY, 22603 Hamburg, Germany \\ R. Carcagno, T. N. Khabiboulline, S. Kotelnikov, A. Makulski, J. Nogiec, R. Nehring, M. Ross, and W. Schappert \\ Fermilab, Batavia, Illinois 60510, USA \\ (Received 1 December 2009; published 9 July 2010)
}

\begin{abstract}
The European x-ray free electron laser is under construction at Deutsches Elektronen-Synchrotron (DESY). The electron beam energy of up to $17.5 \mathrm{GeV}$ will be achieved by using superconducting accelerator technology. Final prototyping, industrialization, and new infrastructure are the actual challenges with respect to the accelerating cavities. This paper describes the preparation strategy optimized for the cavity preparation procedure in industry. For the industrial fabrication and preparation, several new hardware components have been already developed at DESY. The design and construction of a semiautomated rf-measurement machine for dumbbells and end groups are described. In a collaboration among FNAL, KEK, and DESY, an automatic cavity tuning machine has been designed and four machines are under construction. The functionality of these machines with special attention to safety aspects is described in this paper. A new high pressure rinsing system has been developed and is operational.
\end{abstract}

DOI: 10.1103/PhysRevSTAB.13.071001

PACS numbers: 85.25.-j, 29.20.Ej, 84.40.-x

\section{INTRODUCTION}

The European $\mathrm{x}$-ray free electron laser (XFEL) $[1,2]$ is based on a superconducting linac comprising 100 accelerator modules housing eight tesla-type cavities each. The cavity design gradient is $23.6 \mathrm{MV} / \mathrm{m}$.

In preparation for the series production of the European XFEL's accelerating cavities, two final surface treatment schemes [3,4] - electropolishing (final EP) and final buffered chemical polishing (BCP flash) - were studied with cavities from two different vendors. At the same time, the strategy to weld the cavities to their helium vessels prior to the final surface treatment was investigated. As a result, yield curves for the different schemes (with or without helium vessel), yield curves for the different vendors being qualified for the XFEL cavity production [5], and a preparation strategy for the XFEL cavity production became available.

One important step during cavity fabrication is the rf frequency tuning of dumbbells and end groups. In order to considerably shorten the tuning time and thus the cost, a dedicated apparatus was developed [6]. The prototype was successfully used for the recent cavity production. Two more machines are under fabrication with only minor changes. A key issue for the industrial use is automation and documentation.

The finished cavities need to undergo a frequency and field flatness tuning. Dedicated tuning machines for the

*detlef.reschke@desy.de series cavity production were built and are under commissioning [7]. While the mechanical parts were contributed by DESY, the development of software and electronic devices was done at FNAL. Conformité Européenne (CE) certification of the entire machine according to European rules and laws is a must. The final machines can be operated by non-rf experts.

The new high pressure rinsing system [8] implements the system's lay out improvements compared to previously existing high pressure rinsing (HPR) stands. One further design goal is to set up a HPR stand prototype applicable in an industrial production line. After commissioning, the new HPR stand became a standard hardware in use for the cavity preparation processes at DESY.

\section{IMPROVED CAVITY PREPARATION CYCLE FOR INDUSTRIALIZATION}

For the European XFEL, the industrial vendors will do the mechanical manufacturing of the tesla-type cavities as well as the surface preparation. The cavities will be supplied ready for $\mathrm{rf}$ acceptance tests at $2 \mathrm{~K}$, including the complete surface treatment, the individual cavity helium vessel, and all rf antennas.

In a first industrialization step, the main surface material removal by electropolishing (EP) was transferred to industry. In a second step, a proposal for a reduction of preparation steps in order to reduce preparation cost without losing performance of the resonators was presented in 2007 [3]. This proposal was made for the short chemical etching 


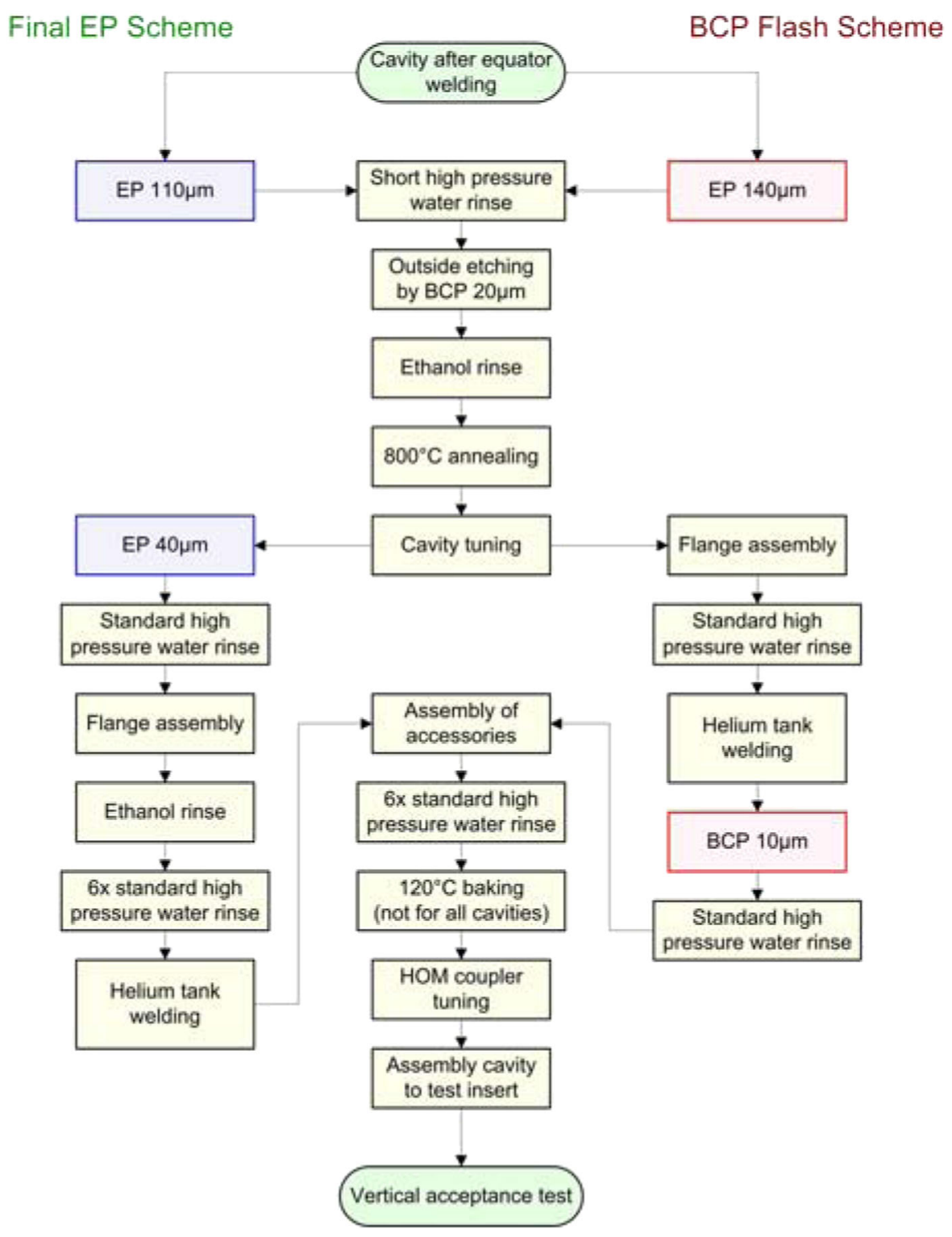

FIG. 1. (Color) Work flow diagram of the optimized preparation cycle for vertical rf acceptance test with He tank welded.

of about $10 \mu \mathrm{m}$ by buffered chemical polishing (BCP flash) as the final surface removal treatment. Now, this concept of reduced preparation steps was transferred to the final surface removal of $40 \mu \mathrm{m}$ by EP (final EP) sequence (Fig. 1) as well. Thirty superconducting resonators, delivered by two industrial companies, have undergone the main EP at two different industrialized EP facilities. The applicability of the proposed optimized preparation cycle is tested on these resonators.

\section{A. Preparation steps}

As a baseline of the optimized XFEL preparation sequence proposal, both for BCP flash and final EP, the helium vessels are welded on at the earliest state possible. The $120^{\circ} \mathrm{C}$ bakeout [Fig. 2(b)] as well as the vertical rf acceptance test at $2 \mathrm{~K}$ are done with cavities already installed into their helium vessels [Fig. 2(a)].

For the final EP sequence, no additional surface preparation, other than 6 times standard HPR (duration $2 \mathrm{~h}$ each), is applied after the helium tank is welded on. The field measurement system [9] is installed right after the final electropolishing and remains inside the resonator until the accessories such as pickup probe, higher order mode $(\mathrm{HOM})$ probes, and $\mathrm{rf}$ input antenna are installed.

\section{B. Main EP in industry}

The main EP was transferred to industry before the start of the preparation test sequences. During main EP $110 \mu \mathrm{m}$ niobium is removed for the final EP sequence and $140 \mu \mathrm{m}$ for the BCP flash. In order to avoid the HPR preparation 


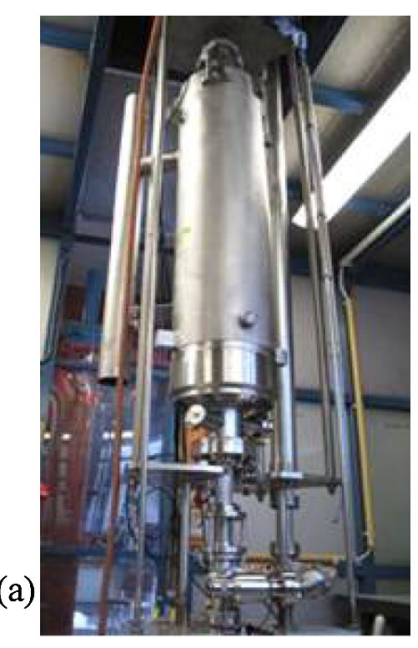

(b)

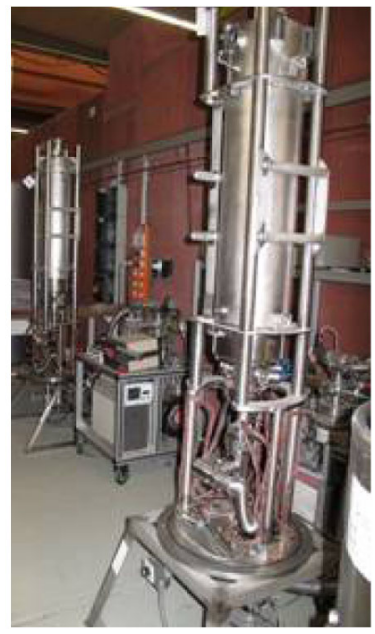

FIG. 2. (Color) (a) Cavity equipped with helium tank and all accessories installed to the vertical test insert. (b) Cavity dressed with helium tank installed to $120^{\circ} \mathrm{C}$ baking apparatus before vertical rf measurement.

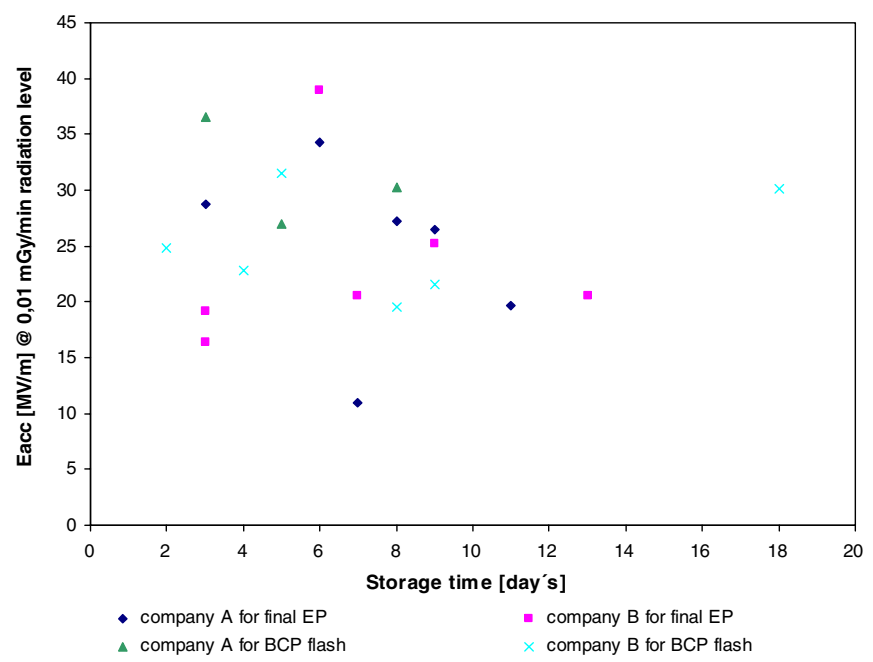

FIG. 3. (Color) Correlation of storage time of cavities in ultrapure water and acceptance gradient at first rf test.

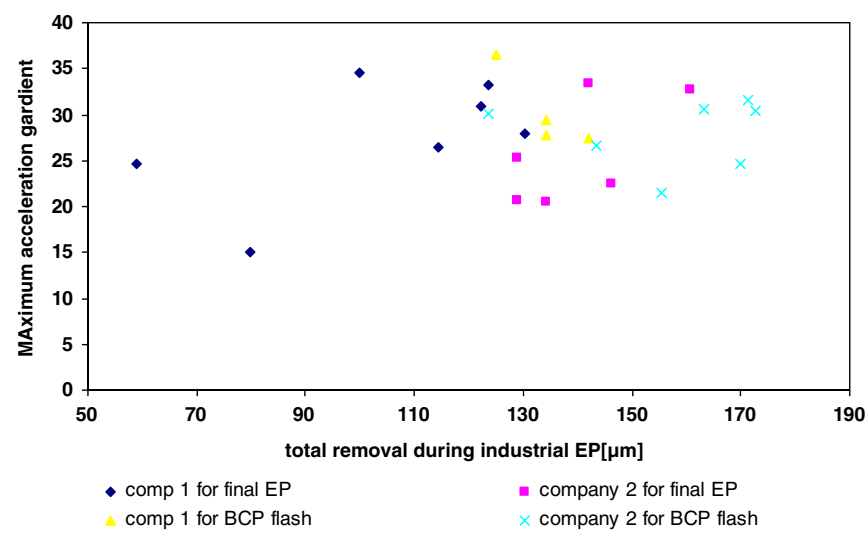

FIG. 4. (Color) Comparison of maximum acceleration gradient and removal of material during main EP treatment at industry. step in industry, the main EP cycle was modified, compared to the former preparation cycle at DESY [10].

For transport to and storage at DESY, the cavities are filled with water of a conductance below $<0.14 \mu \mathrm{S} / \mathrm{cm}$.

For a storage time of less than one week after the main EP, no significant influence on resonator performance is observed (Fig. 3). For EP process steering, one company applies constant voltage, and the second company applies constant current. No differences in cavity performance are observed between these two steering processes.

A comparison of the gradient yield depending on removal of niobium during main EP at industrial EP facilities (Fig. 4) and the main EP at DESY does not show significant differences [5,11].

\section{Cavities available for test}

In addition to the preparation sequences, the main EP at industry had to be tested in parallel. Thirty cavities from production lot 6 , fabricated by two different suppliers, were available for the test sequence. In a first batch, a randomly distributed set of ten resonators each, five from supplier one (CVS1) and five from supplier two (CVS2), was chosen and sent to the two industrial EP facilities (A + B) for main EP treatment. In the second batch of cavities, no random distribution of CVS1 and CVS2 cavities to the two industrial EP facilities took place (Table I).

During the test of these first 20 resonators, three cavities showed low maximum gradient. Two of these cavities were removed from their helium vessels and further investigated. The investigation revealed that these cavities were limited at equator welds or bad spots close to the equator. One cavity showed a $Q$ switch, not related to the preparation sequence. Another cavity showed a leakage to the helium reservoir. The retest is not yet finished. These resonators were excluded from the analysis relevant for the proof of preparation sequences $[5,12]$.

Because of strong heating of the HOM coupler antennas, three cavities were limited during the first power rise. For these cavities, additional HPR was applied after the removal of the HOM probes. The tests were repeated. The final test results for these cavities were included in the statistics of the different preparation sequences.

TABLE I. Distribution of resonators for main EP at industry.

\begin{tabular}{|c|c|c|}
\hline Batch No. 1 & $\begin{array}{l}\text { Main EP @ } \\
\text { company A }\end{array}$ & $\begin{array}{l}\text { Main EP @ } \\
\text { company B }\end{array}$ \\
\hline \multirow[t]{2}{*}{ For final EP (110 $\mu \mathrm{m}$ removal) } & $2 *$ CVS1 & $2 *$ CVS1 \\
\hline & $3 *$ CVS2 & $3 *$ CVS2 \\
\hline \multirow[t]{2}{*}{ For BCP flash (140 $\mu \mathrm{m}$ removal) } & $3 *$ CVS1 & $3 *$ CVS 1 \\
\hline & $2 *$ CVS2 & $2 *$ CVS2 \\
\hline \multicolumn{3}{|l|}{ Batch No. 2} \\
\hline For final EP (110 $\mu \mathrm{m}$ removal) & $4 *$ CVS1 & $4 *$ CVS2 \\
\hline For BCP flash (140 $\mu \mathrm{m}$ removal) & $\cdots$ & $2 *$ CVS1 \\
\hline
\end{tabular}




\section{Accessory, couplers, and antennas}

To minimize the preparation steps following the vertical acceptance test, all accessories such as higher order mode pickup antennas, rf signal pickup probes, and high $Q$ power coupler antennas should be already installed and tested during the vertical test at $2 \mathrm{~K}$. A variable high quality antenna (hQ), which is presently in use for the tests does not allow transportation over long distances. As a consequence, new designs of fixed hQ antennas and HOM coupler probes were installed and tested [13].

For the vertical acceptance test for the XFEL a cavity test in continuous mode operation (CW) at $2 \mathrm{~K}$ is foreseen. The HOM probe design currently in use is designed for pulsed mode operation and capable to run in pulsed mode operation. An improved design of HOM probes for CW operation is under investigation [13] and has been tested in these test sequences as well. Some resonators were limited in performance by these antennas. A further optimization of the design, the preparation of antennas for test, and the test procedure of fully equipped cavities is ongoing.

\section{E. rf test results}

To limit the dark current level of the FLASH accelerator, cavities to be installed into FLASH should not exceed a radiation level (acceptance gradient $=$ Eacpt) of $10^{-2} \mathrm{mGy} / \mathrm{min}$ in the vertical test set up at DESY. The statistical proof of the proposed sequences is based on the test results of 20 four resonators.

Cavities passing the final EP sequence show an average maximum acceleration gradient of Eacc $\max =29 \mathrm{MV} / \mathrm{m}$ and an acceptance gradient of Eacpt $=26.1 \mathrm{MV} / \mathrm{m}$ in the first $\mathrm{rf}$ test after preparation. A spread of maximum gradients from 20.6 up to $38.9 \mathrm{MV} / \mathrm{m}$ is observed (Fig. 5). For the BCP flash cycle the average gradient of Eacc max is 27.7 MV/m. The calculated average value of the acceptance gradient Eacpt is $26.4 \mathrm{MV} / \mathrm{m}$ (Fig. 6). The maximum acceleration gradient of this preparation spans from 20.5 up to $36.7 \mathrm{MV} / \mathrm{m}$.

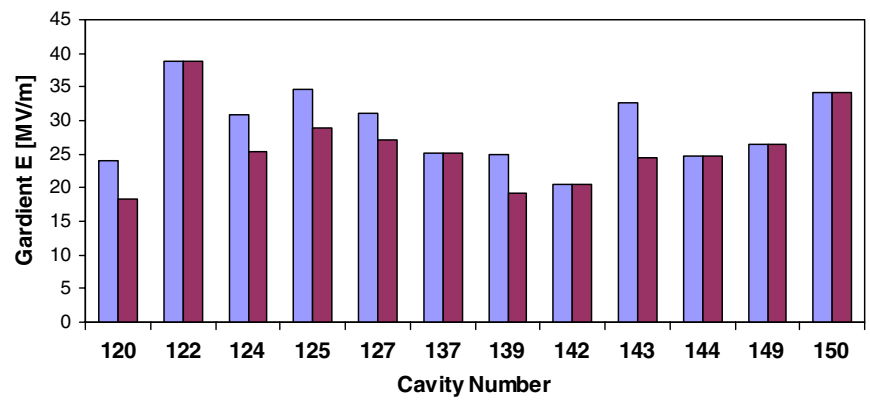

FIG. 5. (Color) Test results of cavities prepared for test according to the "final EP" treatments cycle. Blue: maximum acceleration gradient Eacc; Red: acceptance gradient Eacpt.

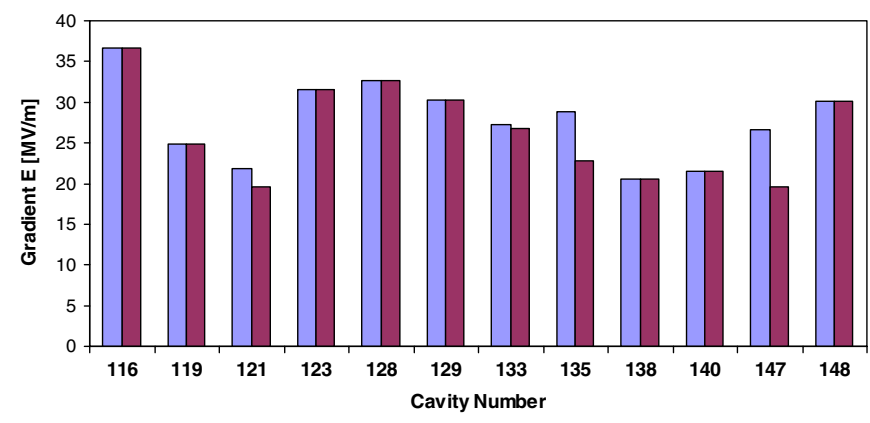

FIG. 6. (Color) Test results of cavities prepared for test according to the "BCP flash" treatments cycle. Blue: maximum acceleration gradient Eacc; Red: acceptance gradient for FLASH Eacpt.

\section{F. Preparation cycles "with He tank" and "without He tank"}

The improved preparation cycle contains the helium tank (vessel) welding as early as possible, i.e. before the vertical rf acceptance test ("vertical rf test with He tank"). In Figs. 7(a) and 7(b) the usable gradient for the first and last rf test of this new scheme is compared to the established procedure, i.e., welding the He tank to the cavity after successful rf test ("vertical rf test without He tank"). The usable gradient is the lowest value among quench gradient, radiation limit, or $\mathrm{rf}$ losses in $\mathrm{CW}$ operation exceeding $100 \mathrm{~W}$.
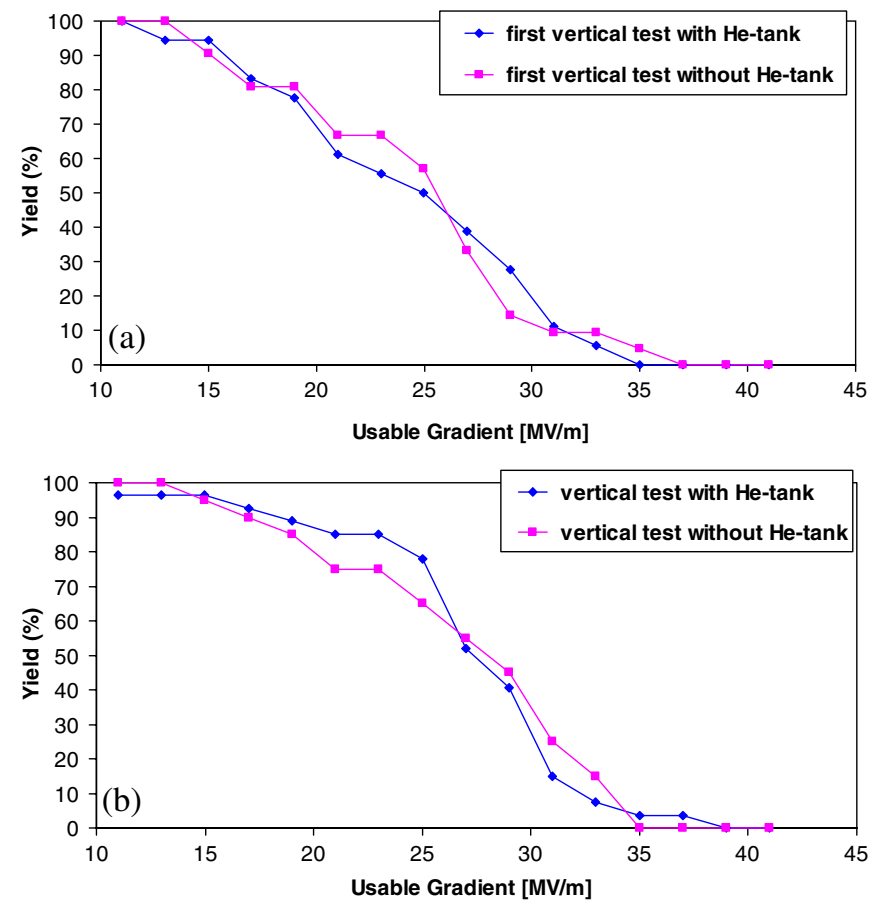

FIG. 7. (Color) Yield plot of usable gradient for first (top) and last (bottom) vertical $\mathrm{rf}$ acceptance test depending on preparation strategy: "vertical rf test with He tank" or "vertical rf test without He tank." 
It is obvious that the results are identical within the measurement error, and the recently optimized scheme can be applied without any degradation in the cavity performance during the vertical rf acceptance test.

\section{DEVELOPMENT AND DESIGN OF A RF-MEASUREMENT MACHINE}

\section{A. Motivation}

The standard half-cell forming method for the production of $1.3 \mathrm{GHz}$ niobium cavities is deep drawing. Precise optical 3D measurements done on deep drawn half cells have shown that a shape accuracy of $0.4 \mathrm{~mm}$ is the realistically achievable tolerance for fine grain niobium.

Deformation will occur again during welding of the dumbbells at the equator due to the high temperature and stress relaxation. The correct shape has to be determined by a frequency measurement. DESY developed a so-called "trimming procedure" to compensate the deviation from the theoretical rf shape [14]. This procedure is used as a quality management tool which guarantees the expected length and resonant frequency of the finished nine-cell cavity.

For the execution of the frequency measurement a simple device (Fig. 8) was built and used for the fabrication of 72 cavities for FLASH.

The fabrication of 800 cavities for XFEL will require about 30000 rf measurements. New arrangements concerning ergonomic and economic aspects are needed.

These facts gave us the motivation to develop and design a rf-measurement machine described below. The rfmeasurement machine was named "HAZEMEMA" as an abbreviation for the German word Halbzellenmessmaschine (English translation: half cell measurement machine).

\section{B. Principle of length and frequency control}

The resonant frequency changes due to shape errors of half cells and welding shrinkage at the equator and iris. The

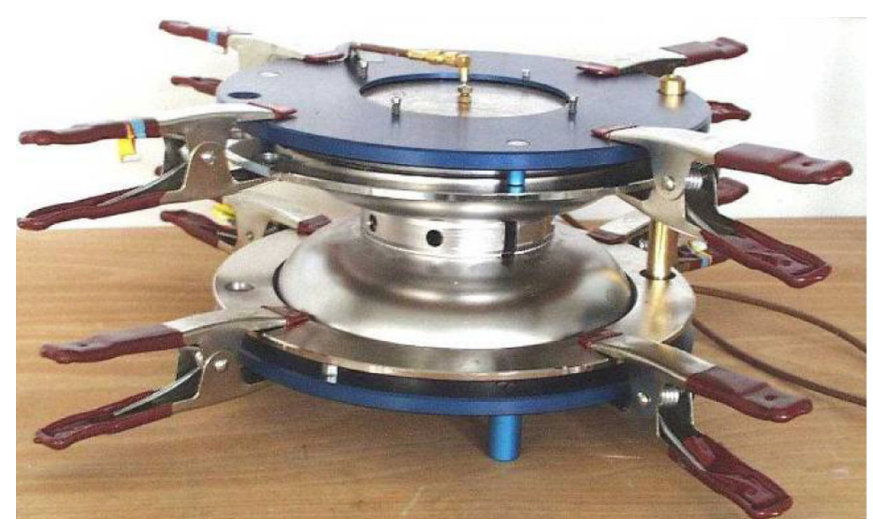

FIG. 8. (Color) Simple rf-measurement device with clamped dumbbell. tuning of the whole cavity to a flat field profile at the correct resonant frequency by deformation of cells in longitudinal direction results in additional errors in length. To achieve both a correct resonant frequency and a correct length, the equators of every dumbbell and end group are trimmed before the equators of a nine-cell cavity are welded by electron beam welding.

For the rf measurement on half cells or dumbbells, the parts are placed between two contact plates (Figs. 8-10). In the center of each plate there is a small antenna connected to a network analyzer (NWA). The resonant frequency in the test setup is compared to the calculated frequency. An over length at the equator and at the iris is included in the calculation. The dumbbell has two resonant frequencies of 0 mode and $\pi$ mode. Equator trimming is calculated from the deviation of the $\pi$-mode frequency and anticipates the cavity tuning after welding. The contact plates are made from niobium to avoid pollution of the test objects before welding. A good rf contact, especially at the equator, is crucial for the frequency measurement. Therefore, clamps are used to press the equator against the contact plate. At the same time, a deformation of the cell has to be avoided. The quality factor of the resonance is used to check the contact between the test objects and the contact plate.

Asymmetry of the dumbbells is measured by comparing the frequency change from a perturbation bead placed through the holes in the contact plates (Fig. 9).

The following quality control steps are applied next: (i) frequency measurement of half cells to check the shape and reproducibility of deep drawing procedure; (ii) frequency measurement of end half cell units to check before and after reshaping; (iii) frequency measurement of dumbbells and end groups to calculate the trimming value; (iv) frequency measurement of dumbbells and end groups after equator trimming as a check before the cavity welding and to sort the positions of dumbbells in the cavity.

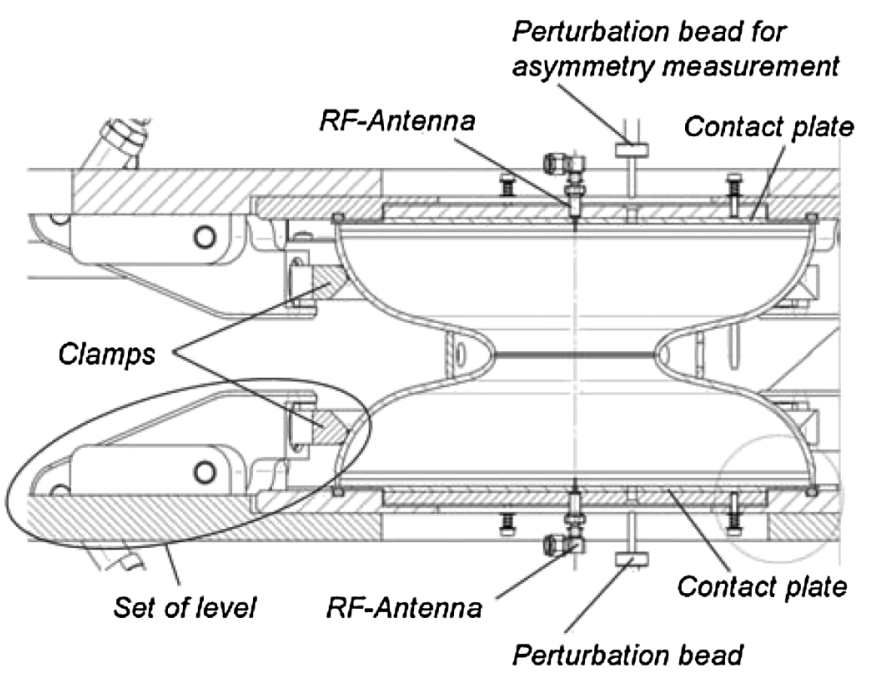

FIG. 9. Covered dumbbell in clamped position. 


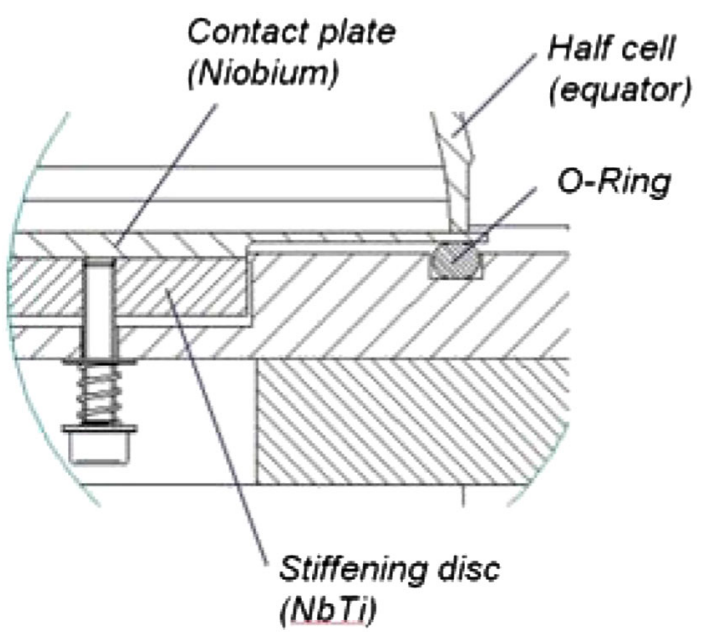

FIG. 10. Detail view of the contact area.

\section{Frequency measurement of half cells and dumbbells}

There are three different contact plates for the measurement of all existing test objects. The contact plates are designed in such a way that deformation of the plates during pressing against the half cell is avoided. The clamping forces are limited for the equator to $\leq 1000 \mathrm{~N}$ and for the iris to $\leq 100 \mathrm{~N}$ (effective force value at the part).

\section{Frequency measurement of end half cells, end half cell units, and end groups}

The shape of the end half cell differs from the shape of a normal half cell. The frequency of an end half cell elongated with a "cutoff tube" should be equal to the frequency
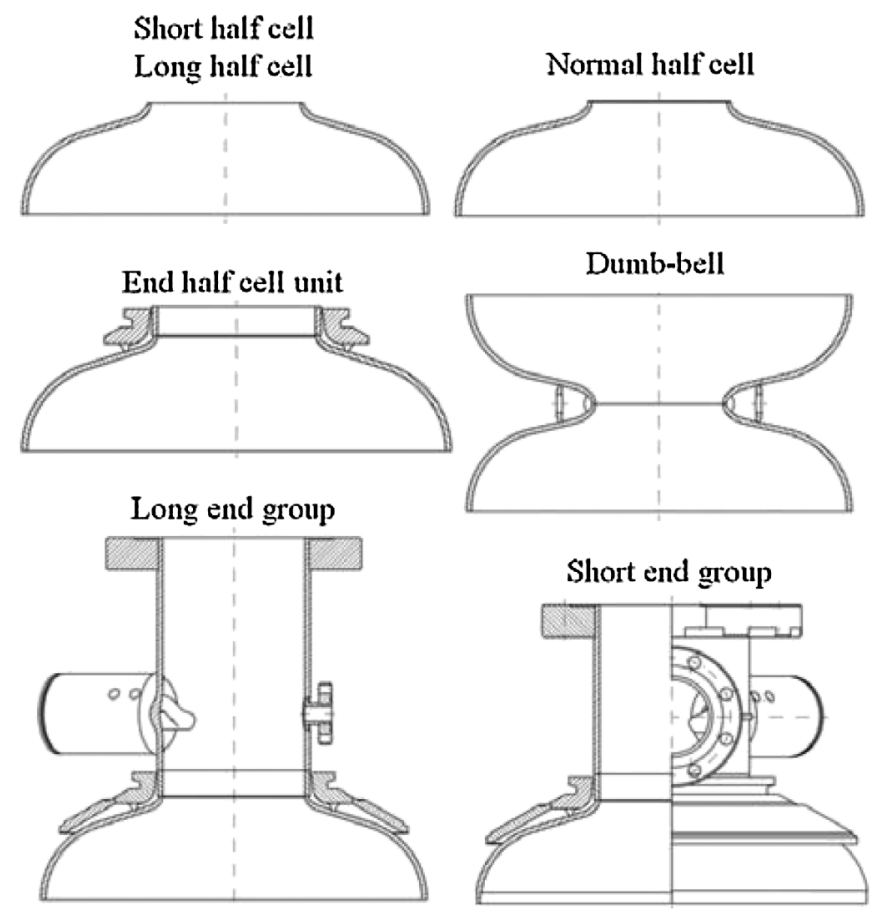

FIG. 11. Measurable parts of the $1.3 \mathrm{GHz}$ nine-cell cavity. of $\pi$ mode. An additional cutoff tube has to be used for the frequency measurement of the end half unit (Fig. 11).

\section{E. Conceptual machine configuration}

The minimum requirements of the machine concept were defined before starting the project. The rfmeasurement machine has to be a portable, robust, and compact test stand for its application near to the fabrication areas, e.g., workshop. It should be equipped with mostly industrial mechanical parts, hardware, and software; "One-person" operation must be possible.

Because of the high number of measurements for a mass production of 800 cavities for XFEL, the test duration has to be substantially reduced compared to the old, simple device. It requires a high grade of automation so that nonexperts can operate HAZEMEMA, too.

\section{F. Development}

In 2006 we started with the development and design of a prototype of HAZEMEMA. After completing the technical drawings, the fabrication and ordering of parts, assembly, and first commissioning took approximately 10 months. In the middle of 2007 the first HAZEMEMA was completed and ready for use.

Since then HAZEMEMA was used during the fabrication of 41 cavities for FLASH.

As the machine fulfilled all requirements and because of the good experiences in using HAZEMEMA, two more machines were ordered in 2008. DESY will provide the manufacturer of the European XFEL cavities with these machines.

\section{G. General design}

The general requirements are based on the conceptual configuration and on the use of the existing contact-plate system (Fig. 9) from the old manually operated device.

\section{H. Mechanical design}

The mechanical design was done by using the 3D-CAD system SOLID EDGE (Siemens PLM Software). A simulation of the motion sequences was done to avoid collision damages during clamping movements. The rf measurements on six different parts and subgroups (Fig. 11) summarized as test objects-of the $1.3 \mathrm{GHz}$ nine-cell cavity must be realized.

\section{Clamping and contact-plate system}

It was a special task to realize full automated clamping of the test objects with appropriate electrical contact but without deformation of the test objects nor the contact plates. A deformation during $\mathrm{rf}$ measurement must be avoided because it would give an unacceptable error of the frequency measurement. The solution to the problem 


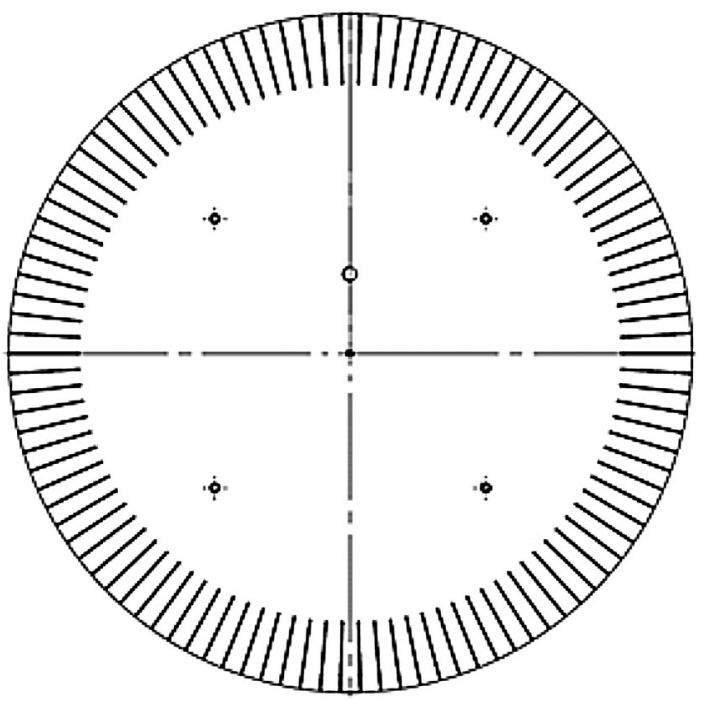

FIG. 12. The top view of the contact plate showing 107 slots in the equator area for sufficient electrical contact.

was the use of well-defined clamping forces and applying the force at the right position.

Covering of the rf volume is done with niobium disks which were multiple slotted in the contact area (Fig. 12) to ensure good electrical contact in the case that the equator surface is not planar. The contact plate is supported by a spring system to avoid deformation from its own weight.

\section{J. Pneumatic system}

The first design of the pneumatic circuit, system concept, and the main linear actuator was done by the company FESTO using standard components of the FESTO product line.
During assembly and testing of the prototype, we redesigned the pneumatics for a better handling and for safer pneumatic movements.

\section{K. Safety interlock}

High forces and clamping speed develop in this machine when niobium test objects are pressed.

Therefore, it is necessary to minimize pinching hazards for the operator. For this reason, a safety interlock was manufactured by DESY. This system was built according to all valid European regulations, like VDE, EN, and CE guidelines.

All electrical parts are established and proven. These were supplied by well-known manufacturers.

\section{System controllers}

The system/machine controllers consist of the following parts (Fig. 13): (i) The main controller is a PC, running GNU/Linux OS (Ubuntu ${ }^{\mathrm{TM}}$ by Canonical Ltd.); (ii) the slave controllers are two programmable logic controllers (PLCs) from FESTO named analog and digital PLC, respectively, because we separated the input/output (IO) channels into these two categories; (iii) a motor controller from Phytron Elektronik GmbH; (iv) the NWA measuring the resonance frequency and the quality factor $(Q)$; (v) the safety controllers, which observe the closed cabinet (Fig. 14) during measurement and also cut off the power, if the emergency-off button is pressed.

The Phytron ${ }^{\circledR}$ motor controller controls the movable upper clamping system (Fig. 14), its position, speed, acceleration, and motion stop in the correct position. The initialization is done only once, when the plate is driven to the home switch. After initialization, the main controller

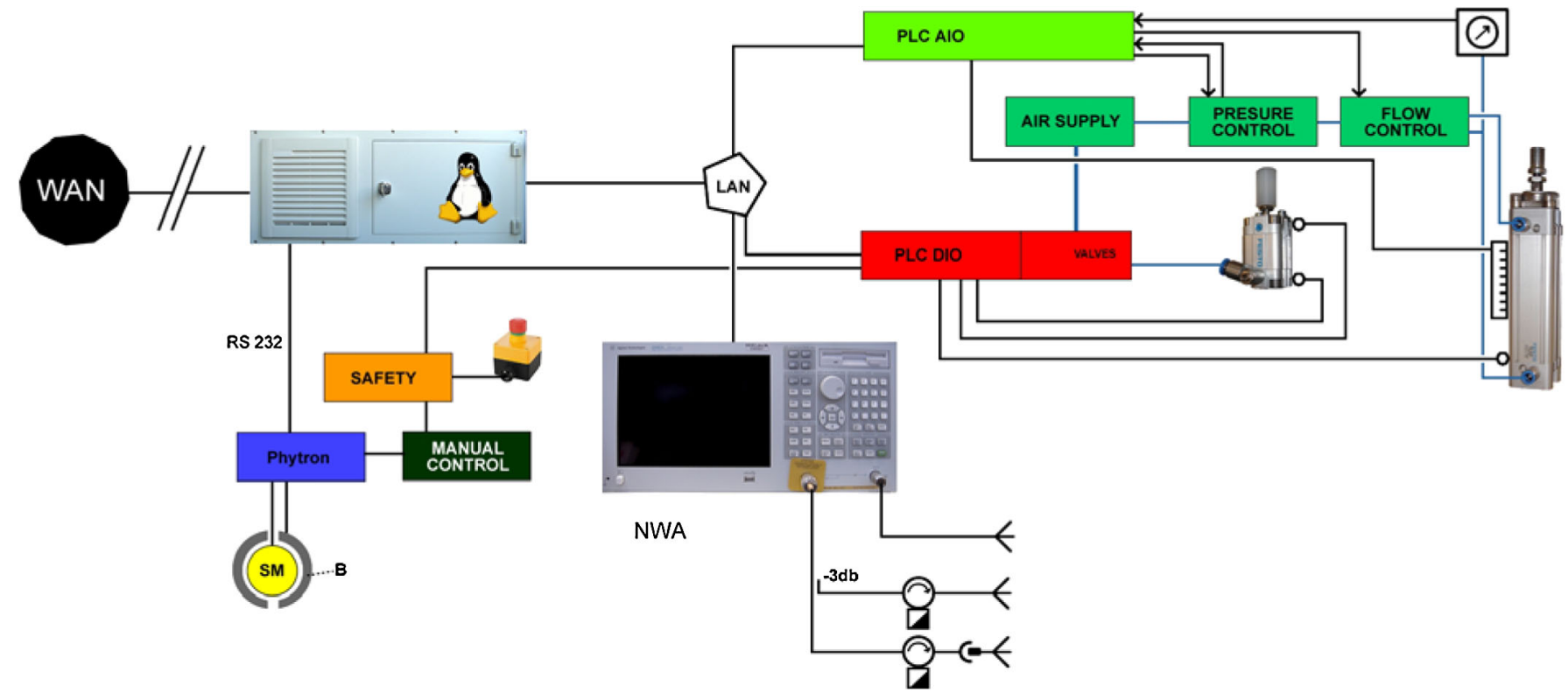

FIG. 13. (Color) Block diagram of HAZEMEMA control system. 


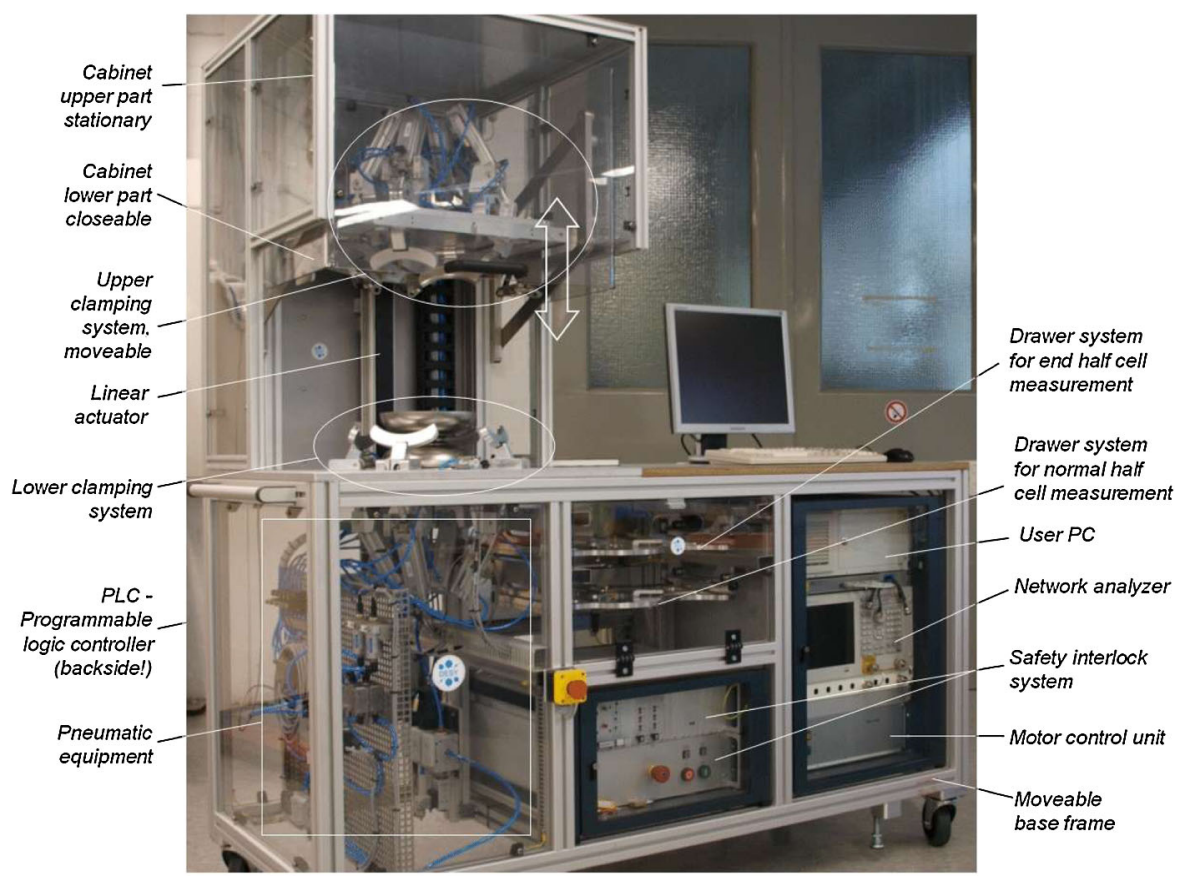

FIG. 14. Prototype rf-measurement machine (called HAZEMEMA).

sends the command where the plate should be driven to. Because of various test object dimensions, there is no predefined position for stopping. The virtual target is the maximal drivable range of the plate and the stop command is generated by a sensor signal (light barriers or force detectors), selected by the digital PLC.

The analog PLC is running in remote-IO mode, meaning that all IO channels (analog input/output with 0/4-20 mA signal) are accessed by the main controller directly via Ethernet. The sensors are pressure detectors and position transmitters, used on all pneumatic cylinders, where the plunger usually does not reach its final limit. These are all clamp cylinders and the front actuator, which centers the test object before it is clamped. The clamp cylinders are fed with air pressure. Pressure and flow can be adjusted; therefore, the analog outputs of this PLC are used. For safety reasons the power of these PLC actuators will be cut off if the cabinet (Fig. 14) is open or the measurement procedure for an object is finished.

The digital PLC is running in PLC-controller mode, which permits full remote-IO access in parallel. For this mode, the IO address needs to be configured by hand, in order to be compatible with the remote-IO mode. All digital sensors (except the motor home position and the safety switches) are connected to this PLC. These are mainly: (i) position switches from pneumatic cylinders: (ii) force detectors; (iii) some status bits from the safety unit.

The pneumatic valves are directly built in the digital PLC unit (for the test object positioning/centring actuators and the electrical field disturbance). The load voltage of this PLC will be switched off only by the emergency trip system (not by opening the cabinet). The reason to run this PLC as PLC controller is to select only some of the "end switch" sensors for the motor controller. In parallel, the machine status, shown as a traffic light, is also managed by the logic in this PLC, making it independent from the main running program.

The main controlling program is designed in LABVIEW $^{\text {TM } 1}$ software, running on GNU/Linux OS $\left(\mathrm{Ubuntu}^{\mathrm{TM}}\right)$. This computer contains two network interfaces; one is used to connect the machine to the outer world, where it takes the IP numbers via dynamic host configuration protocol (DHCP) and will be accessible via the secure shell protocol ssh (for maintenance) and the protocol http (to access the measured data). The second network interface is used as local machine network for the PLCs and the NWA. The computer itself acts as DHCP server for the network devices and uses EASY-IP (UDP protocol from FESTO) for the PLCs, VISA ${ }^{\mathrm{TM}}$ software for the data access and a virtual network computing (VNC) software (REAL VNC under GNU general public license) for the maintenance of the NWA. The motor controller is connected to a RS232 interface.

The LABVIEW ${ }^{\mathrm{TM}}$ software program is separated into state driven subgroups for: (i) the test object positioning/

\footnotetext{
${ }^{1}$ LABVIEW $^{\mathrm{TM}}$ and $\mathrm{VISA}^{\mathrm{TM}}$ are trademarks of National Instruments. This publication is an independent publication. National Instruments is not affiliated with the publisher or the author, and does not authorize, sponsor, endorse, or approve this publication.
} 
centering system-which enables the back actuators and moves the test object against them using the front actuator; (ii) the lower (fixed) clamping system-adjusting clamp force and speed of the clamps and checking that the active clamp is finally no longer moving (thereby the resonance frequency in the object should be stable); (iii) the upper (movable) clamping system-the functionality is identical to the lower clamping system; (iv) the electrical field disturbance-controlling a plastic stick in the table and in the movable contact plates; (v) the linear vertical actuator system - controls the motor, enables end switches (e.g., light barriers), and checks the mounted contact-plate type; (vi) the auxiliary part-where the main air and power will be observed; (vii) the NWA part-it finds the resonances in a wide frequency range, reduces the span, and finally measures each resonance frequency and $Q$ value.

In order to use the machine in a normal fabrication process, some data exchange is needed. At the moment it is possible to use a secure copy protocol or a memory stick in order to transfer the object names and parameters to the machine and to get the measurement results back from it. For the final version it is planned to use a local web server to access the measured data (and maybe also to upload the object names and parameters). Thus, the operator only needs to select the right object, which is inserted for the measurement. Alternatively, it is always possible to enter the data by hand prior to measurement, and conventionally read the data from the screen.

\section{Experiences during cavity fabrication}

The prototype machine was used for the DESY cavity production lots 6,8 , and 9. A total of 41 cavities were fabricated by using HAZEMEMA. More than $1600 \mathrm{rf}$ measurements were done.

HAZEMEMA was used without restriction of any kind by one operator. The test duration could be considerably reduced compared to the manual operation (see Table II).

\section{N. Improvements}

After commissioning and first use of the prototype some improvements were done.

(i) In the previous clamping system the clamps (Fig. 9) were equipped with a soft ring to avoid damages on the niobium test objects. Because of the fact that this type of ring absorbed too much of the clamping forces, it was removed.

TABLE II. Test duration.

\begin{tabular}{lcc}
\hline \hline Test object & $\begin{array}{c}\text { Decreased } \\
\text { test duration }\end{array}$ & $\begin{array}{c}\text { Realized test } \\
\text { duration (min) }\end{array}$ \\
\hline Half cell & $67 \%$ & 1 \\
Dumbbell & $80 \%$ & 2 \\
End half cell unit & $67 \%$ & 1 \\
End group & $67 \%$ & 1 \\
\hline \hline
\end{tabular}

\section{c $\epsilon$}

FIG. 15. (Color) Official label for CE marking.

(ii) Because of unexpectedly high clamping force losses due to the chosen design, it was not always sufficient to apply adequate electrical contact. Therefore, the set of levels (Fig. 9) was optimized and together with a redesign of the pneumatic system the theoretical value of the clamping forces was increased by about $20 \%$ without deforming the test objects.

\section{O. EC conformity operation}

Because the rf-measurement machine will be used externally, i.e., delivered to the cavity fabrication companies by DESY, it has to comply with essential health and safety requirements and it has to be designed, built, and documented in accordance to the machinery directive 2006/42/ EC of the European Community (EC). The so-called "conformity assessment procedures" are mainly consisting of the execution and documentation of risk assessment, creating a user manual, and a formal declaration of " $\mathrm{CE}$ conformity" ( $\mathrm{CE}=$ Conformité Européenne). At the end of the procedure each machine gets a unique serial number and can be "CE certified" by marking it with the official label (Fig. 15).

\section{MECHANICAL DESIGN OF AUTOMATIC CAVITY TUNING MACHINES}

\section{A. Motivation}

For a $\beta \approx 1$ multicell SRF cavity, the fields in adjacent cells must be $\pi$ radians out of phase with each other and the particle must cross a cell in one-half of an rf period. This flat field profile is achieved when the cells are properly tuned relative to each other and the cavity frequency is equal to the design frequency (e.g., $1.3 \mathrm{GHz}$ for XFEL cavity at $2 \mathrm{~K}$ in vacuum). In addition, the tuned cavity must meet dimensional tolerances such as length, straightness, and concentricity of cells.

Cell-to-cell tuning is usually accomplished by slightly deforming every cell plastically until the desired cell frequency is achieved. This procedure is normally foreseen for every cavity after fabrication or after relevant preparation steps like etching or deformations caused by heat treatments or welding. Typical tuning methods [15] are based on special tooling to obtain plastic deformation of every cell manually by an expert operator. This is a very time-consuming procedure. It is not adequate for cavity series productions needed for SRF-based projects like the XFEL. An alternative method is shown by the semiautomated prototype tuning machine which has been used at DESY for several years. The function of the machine is 
based on three independent vice units to squeeze or stretch a cell while keeping the cavity straight.

The major principle of this machine is the basis for a new series of high level automation tuning machines developed within the collaboration of FNAL, KEK, and DESY. As for the prototype machine a reliable field flatness of better than $98 \%$ is aimed for after the tuning process.

The use of the machines is not limited to laboratories and institutes. It is foreseen to operate them at industry. They have to be operable by non-rf experts.

Within the collaboration all four machines were mechanically designed and fabricated by DESY including all actuators and sensors for the new automation concepts based on experience with the operating machine. FNAL is providing four completely improved control sets with electronic racks, operating stations, and measurement techniques including all control system hardware and software. The totally renewed software including calculation algorithms by FNAL is an important contribution to the aimed objectives.

Presently, the machines are under commissioning.

\section{B. Overall concept}

The main component of the new entire tuning machines (Fig. 16) is the tuning frame (TF) which accommodates all mechanics, such as vice units, sensors, and actuators needed for plastic deformation of cavity cells. After several optimizations the "frame" became an aluminum alloy cast plate.

All components of the tuning machines are mounted to the $5.2 \mathrm{~m}$ long base frame (BF). It consists of several aluminum alloy extruder profiles. This guarantees high modularity and decreases the weight of machines.

A major component for feedback in tuning procedure is the eccentricity measurement device (EMD). With 11 linear distance generators and two laser sensors it not only gives information on concentricity of single cells to cavity

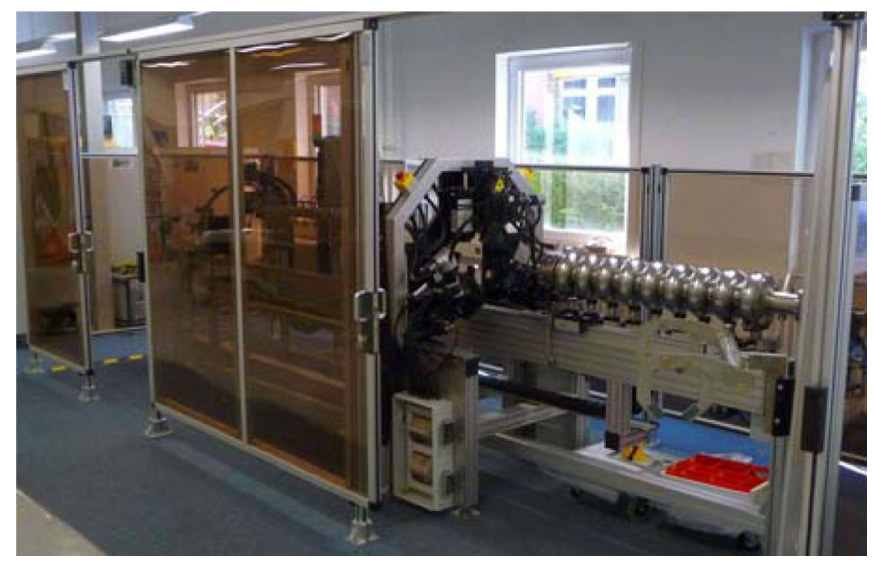

FIG. 16. (Color) Entire cavity tuning machine with machine housing. The door at the cavity insert position is open. axis, but it also displays the length of cavity and perpendicularity of reference planes, of end flanges, and of beam tube flanges.

The measurement of field flatness is done with a bead pull system (BPS). Because of the total length of the BF and to reduce the oscillation of the bead, the system is not a string loop, but a string pulled from a reservoir. The network analyzer (NWA) is connected to the cavity via rf cables in a cable guiding system.

A completely new designed device of the machines is the cavity alignment tool (CAT). It gives the possibility to measure (qualitatively) the direction of cavity deformation compared to its straightness during the tuning procedure.

During all steps of the tuning procedure the cavity is kept and moved by a cavity train (CT) with several supports and lift units which bring the cavity into a defined position for eccentricity measurement.

For safety reasons the entire machine is secured by a machine housing with safety gates. To implement this, the tuning procedure needs to be optimized to a minimum of hand operation to decrease the number of entries

\section{Tuning frame}

The tuning frame (TF) (Fig. 17) is designed modularly. All assemblies including electronic enclosures are mounted to an aluminum alloy cast baseplate. The plate itself only captures the weight of subassemblies. The entire design is done in a way that no counterforces are applied to the baseplate. This design allows assembling and disassembling the whole TF from the machine. Thus, easy assembly and disassembly and, therefore, easy transport is possible. In addition the open structure of the TF allows easy maintenance.

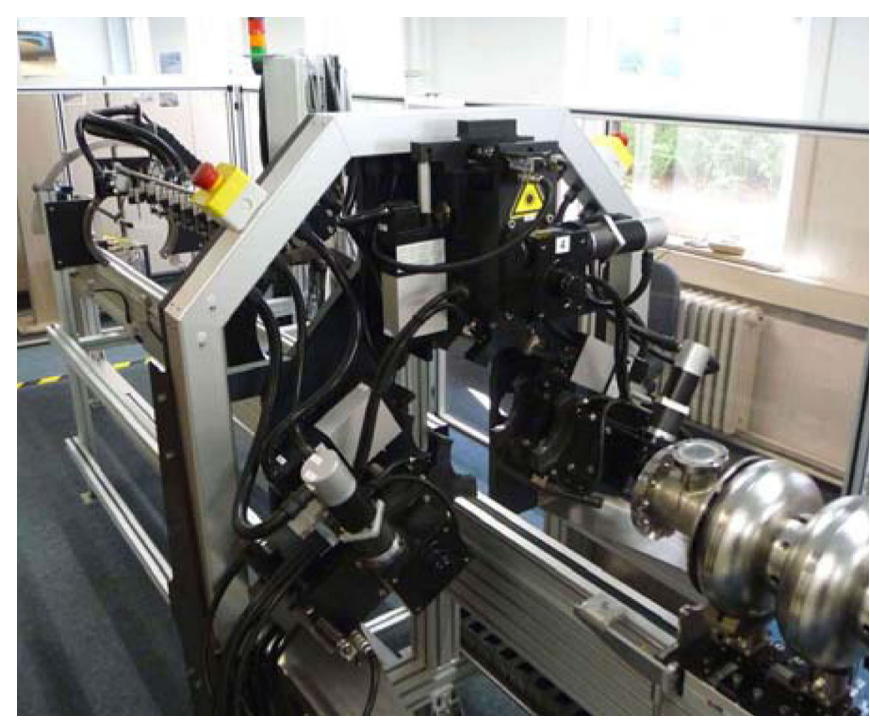

FIG. 17. (Color) The picture shows the tuning frame with the baseplate and the three vise units. On the cable channel the emergency switch is assembled. 


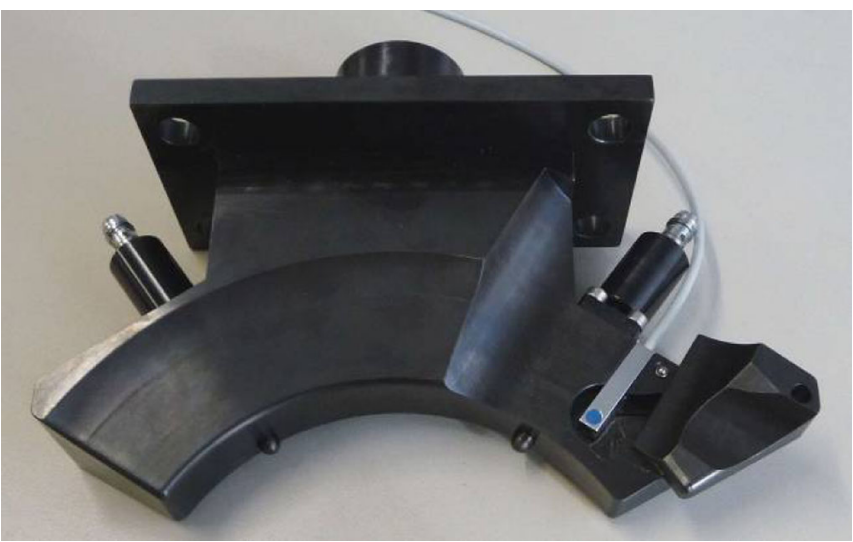

FIG. 18. (Color) Tuning jaw with exchange part and proximity switches.

The tuning frame consists of three identical vise units, arranged in a $120^{\circ}$ angle. Each vise unit has two tuning arms mounted on a rotary axis. Via a stepper motor and two gearboxes, the distance between the arms can be changed for squeezing or stretching cavity cells. The tuning force can reach $90 \mathrm{kN}$ per vise unit. Both arms are free to move around the rotary axis. They are kept in place by low prestressing spring cradles.

Fitted to the tuning arms are special tuning jaws (Fig. 18) with two proximity switches and, in two cases, additional sensors for exchange parts. These exchange parts are removed for end-cell tuning and added for normal cell tuning of XFEL cavities. At the end cells the available space is limited by the HOM coupler ports and main coupler ports. Therefore, the exchange parts are necessary in order to allow end-cell tuning without changing complete jaws. The jaws are, restricted to an angle of a few degrees, free to move around their fixing stud. The complete vise units are moved towards the cavity with linear actuators. This elaborate system including additional protective shields and connection rings (not shown in figures) allows positioning of the vise units at the cavity in a way that the cavity stays free to move a little.

In summary, the cell under tuning in the frame is free to move in every axis during plastic deformation. There is no reaction force additionally brought to the cell. The G forces, caused by the weight of the cavity itself, are compensated by special supports (Fig. 19) of the cavity train (CT supports). Applying this technique it is possible to deform different areas of a cell deviant. This allows tuning a cavity straight independent of its initial state.

\section{Base frame}

To keep the machines modular during development phases and to allow a number of shipments and commissioning at different locations, all components of the entire tuning machines are mounted to a base frame consisting of several aluminum alloy extruder profiles. Compared with

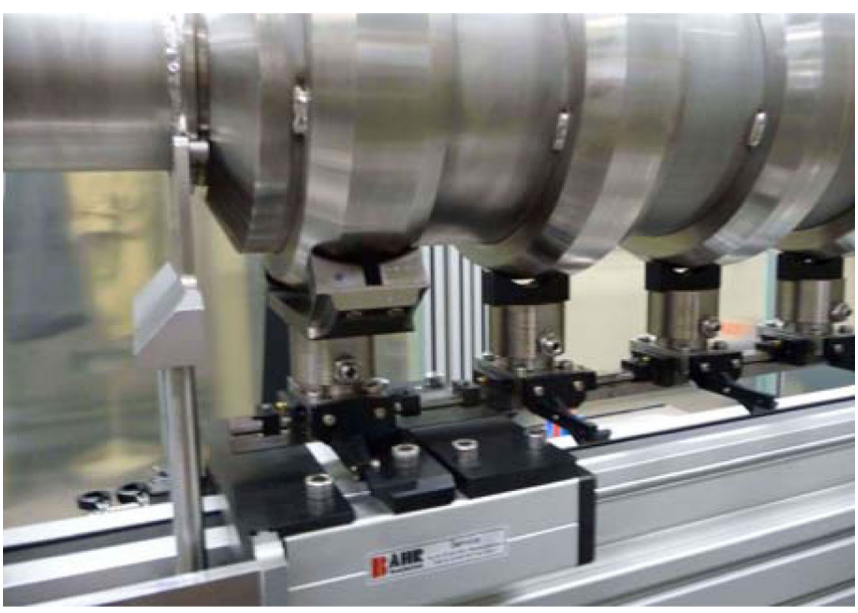

FIG. 19. (Color) Lift units and CT supports with the lift units at the left, contacting the reference plane. A dummy cavity is used to calibrate the eccentricity measurement device.

the prototype machine developed 15 years earlier, the total weight was decreased by $70 \%$.

The machine design is divided into two major assemblies: (i) the tuning frame (TF) with all needed actuators and electronic enclosures; (ii) he base frame including EMD, cavity train, and bead pull system.

\section{E. Eccentricity measurement device}

For the entire tuning process, feedback of mechanical properties such as length and straightness of cavity and concentricity of the cells is very useful to achieve a good tuning result.

All measured mechanical values of the cavity are referred to the cavity reference planes on the connection end flanges. Furthermore, these reference planes are used to align the weld ring and bellow of the helium vessel to the cavity. The cavity alignment in the module cavity string is also performed with the help of these reference planes.

In the eccentricity measurement device (Fig. 20), the cavity is brought into a given measurement position by two lift units. This is done automatically without additional manual operation. The lift units (Fig. 19) are self-adjusting and compensate length tolerances of the cavity. They contact the cavity at the reference planes. Concurrently, the CT supports with adjustable spring lifters compensate the sagging of the cavity caused by its own weight and other forces.

The whole measurement procedure is accomplished by nine distance generators, two flange transmitters, and two laser sensors. The cell distance generators are aligned to the middle of each cell by an automated mechanical system. The middle locations of equator diameters are measured with high resolution potentiometric linear distance generators. A similar system with identical sensors is used to acquire the locations of centers from the beam tube flanges. The length and perpendicularity of the reference 


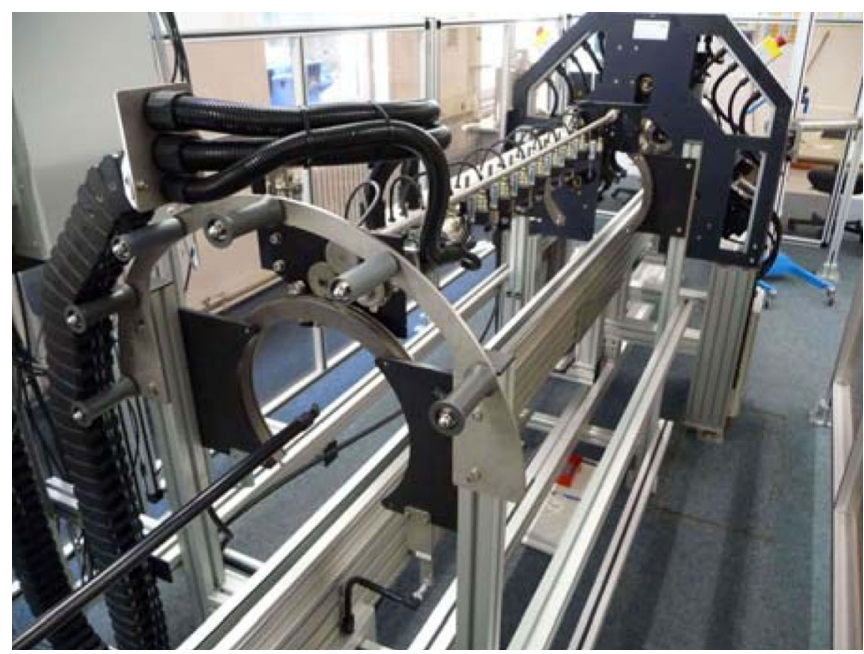

FIG. 20. (Color) Eccentricity measurement device, showing the rotatable arm with the distance generators.

plane is calculated by triangulation of different distance values measured with a precision laser distance detector.

In order to assure a precise measurement, the EMD has to be calibrated with a dummy cavity from time to time (Fig. 19). The dummy cavity made of stainless steel was fabricated with very low tolerances in the range of $\pm 0.01 \mathrm{~mm}$. The actual values of length, cell diameters and location, concentricity, and perpendicularity of reference planes were surveyed by a special 3D measurement device. These values give the reference for the eccentricity measurements. Therefore, for high accuracy measurements it is necessary, that the actual values are included in the calculation algorithms implemented in the tuning software.

In other known measurement devices the sensors are fixed and the cavity is rotated around the beam axes on reference planes. However, in the EMD for the new tuning machines a measurement bar with all distance generators rotates around the cavity, guided on a high precision backlash-free gear wheel. Another new feature is the movement of the measurement bar towards the cavity. The bar is driven automatically by a spline curve gear supported from high prestressed springs.

\section{F. Bead pull system}

The obvious difference between normal BP systems and the one developed is the way the string is kept and tensioned. Normally the string is in a loop. In such systems the string tension is originated by a spring between the ends of string. Because of the string length and elasticity, there is a very high possibility of bead oscillations in lateral and perpendicular directions. In addition, the sagging of the more than $5 \mathrm{~m}$ long string might be a problem.

To avoid these difficulties and to decrease the manual effort, the string is kept open. At the beginning of the tuning procedure the string is pulled from a reservoir (Fig. 21), which at the same time tenses or retards the

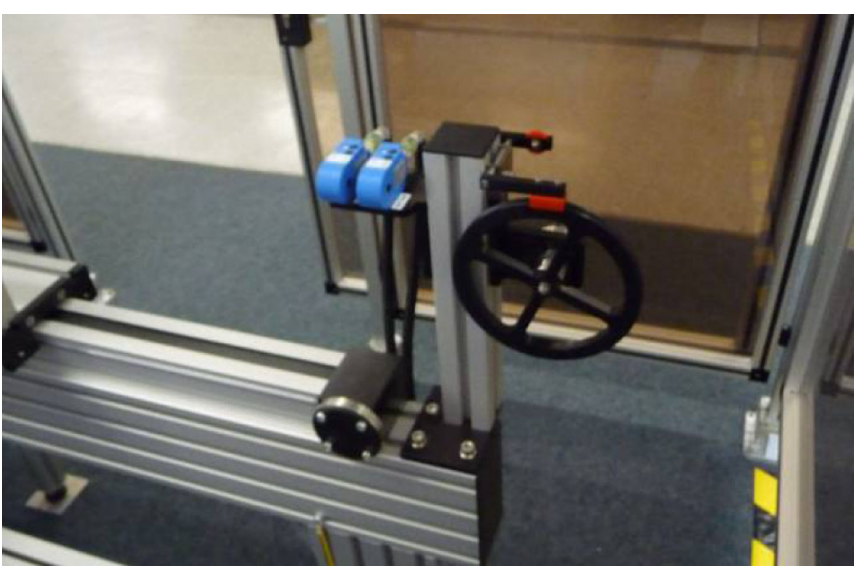

FIG. 21. (Color) BPS string reservoir with sensor unit.

string to an actuator wheel with a motor unit on the opposite site. The very long bead movement of about $5 \mathrm{~m}$ is translated into a short $1 \mathrm{~m}$ vertical movement of the weight by a gear (Fig. 22). This also guarantees a constant string tension.

The field flatness measurement is done several times during the tuning procedure at different cavity locations. Therefore, it is necessary to know the position of the bead and also the position of the cavity to identify relative position of bead in a certain cavity cell. This acquisition is done by several step encoders of actuators and positioning systems.

Before the measurement starts, the initial bead position detection is done by a system of two inductive sensors, which are able to detect the presence of the very small stainless steel bead (tube: $0.2 \mathrm{~mm}$ diameter/ $10 \mathrm{~mm}$ length).

\section{G. Cavity alignment tool}

Beyond field flatness and concentricity, the length and perpendicularity of cavity is a major item for cavity string assembly and thus for cavity tuning.

For series cavity tuning machines a tool to observe the cavity straightness during the tuning procedure of a single

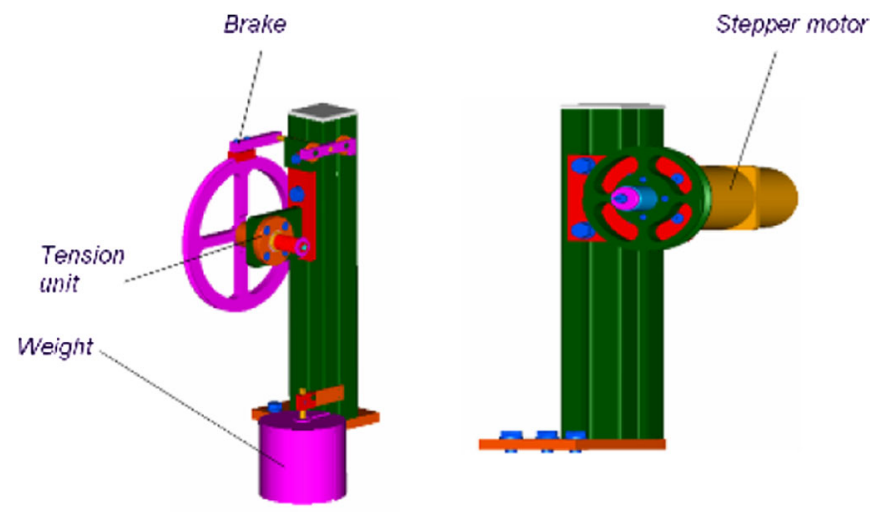

FIG. 22. (Color) BPS string reservoir left and motor unit right. 


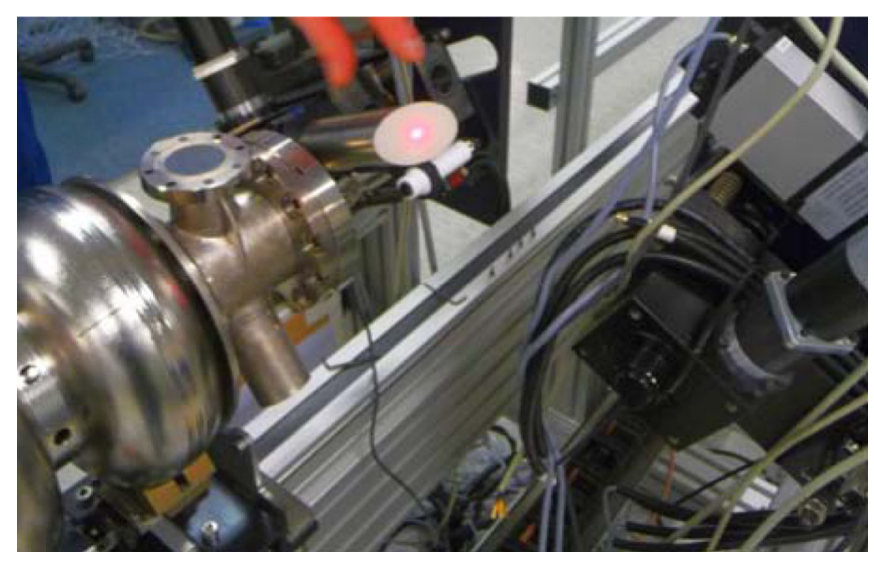

FIG. 23. (Color) Cavity alignment tool, laser spot on target.

cell is needed. In our case a laser beam is sent from the short side beam tube flange to a mirror mounted at the long side beam tube flange of the cavity. Both mirror and laser are easily adjusted during installation to the cavity. The reflected laser beam is sent to an elliptical target fixed to a $45^{\circ}$ notched tube, similar to a periscope (Fig. 23). The target is evaluated by a small industrial camera system.

Through digital image processing and a calculation algorithm, additional deforming distances of single vise units are calculated and the tuning process is concurrently observed.

\section{H. Safety concept}

Since the cavity vendors will be supplied with the tuning machines by DESY, the machines have to comply with essential health and safety requirements and have to be designed, built, and documented in accordance to the machinery directive 2006/42/EC of the European Community (EC). The so-called "conformity assessment procedures" mainly consist of the execution and documentation of risk assessment, creating a user manual and a formal declaration of "CE conformity." At the end of the procedure each machine gets a unique serial number and is marked with the official "CE certified" label.

\section{NEW HIGH PRESSURE RINSING SYSTEM}

\section{A. Motivation}

There are several HPR systems operating successfully worldwide. They are in use for single and multicell cavities and differ in their individual design. All systems share the general common layout, consisting of an ultrapure (UP) water plant supplying the HPR water, a pump to pressurize the ultrapure water, a filter unit to hold back particulates, a cane holding the spraying nozzle, and the motion system to guide the water jets from the nozzles towards all parts of the resonator surface. Most commonly piston or diaphragm based pumps are in use to set up the water pressure. These pumps are often oil lubricated. This design bears the risk that there is a direct pass of oil to the UP water in case of diaphragm or seal failures. In addition, the nonlinear movement of the pistons introduces pulsation to the water jet and can lead to vibrations of the spraying cane. For vibration reduction, damping elements are introduced into the high pressure feed line to the cane.

At DESY a new HPR system had to be set up to increase the turn around time of cavities in the ongoing cavity preparation and to have a back-up system if one HPR stand fails. In this new HPR system the experiences of the past years and improvements in view of an industrialization of the HPR process were included.

\section{B. New HPR system}

The new HPR system (Table III) had to be added to the existing clean room and infrastructure. A clean room cabinet with ISO 4 air quality (Fig. 24) and a buffering UP water (UPW) are added as annexes to the DESY clean room. The new HPR stand is accessible from the ISO 4 assembly area of the existing clean room. To prevent strong variations of the UP water system, a buffer tank of 5001 capacity with an independent UP water system for stand-by operation is integrated into the DESY UP water loop.

TABLE III. Overview on hardware installed in the new HPR.

\begin{tabular}{|c|c|}
\hline \multicolumn{2}{|l|}{ HPR pump } \\
\hline HPR pump type & $\begin{array}{l}\text { Turbine type Sunflo® } \\
\text { P3000 by Sundyne Corp. }\end{array}$ \\
\hline Pressure & 30-100 bar \\
\hline UP water volume@100 bar & $1.2-6 \mathrm{~m}^{3} / \mathrm{h}$ \\
\hline UP water temperature: & $\begin{array}{l}20-80 \mathrm{C} \text { by presetting on } \\
\text { program start }\end{array}$ \\
\hline Spray head & $\begin{array}{l}\text { Standard DESY spray head } \\
8 \text { nozzles }\end{array}$ \\
\hline Turbine bearings & $\begin{array}{l}\text { Whole metal bearing } \\
\text { lubricated by UPW }\end{array}$ \\
\hline Turbine material & Stainless steel \\
\hline $\begin{array}{l}\text { Surface quality of turbine } \\
\text { Motion system }\end{array}$ & polished $\mathrm{Ra} 0.2 \mu \mathrm{m}$ \\
\hline Vertical motion drive & stepping motor + spindles drive \\
\hline Bearings for vertical motion & $\begin{array}{l}\text { Linear bearing hidden } \\
\text { behind separating }\end{array}$ \\
\hline Rotation drive & $\begin{array}{l}\text { stepping motor }+ \text { Teflon }{ }^{\circledR} \\
\text { timing belt }\end{array}$ \\
\hline Rotational drive bearing & $\begin{array}{l}\text { Ceramic stainless nonlubricated } \\
\text { central bearing }\end{array}$ \\
\hline \multicolumn{2}{|l|}{ HPR cabinet } \\
\hline Cabinet air quality & ISO 4 and better \\
\hline Air speed control & $0.15-0.6 \mathrm{~m} / \mathrm{sec}$ variable \\
\hline Air distribution & $\begin{array}{l}3 \text { filter fan units automatic } \\
\text { pressure drop control }\end{array}$ \\
\hline Cabinet material & Stainless steel \\
\hline
\end{tabular}

${ }^{\mathrm{a}}$ Timing belt by Wilhelm Herm. Müller GmbH \& Co. KG, Postkamp 14, D 30159 Hannover, Germany. 


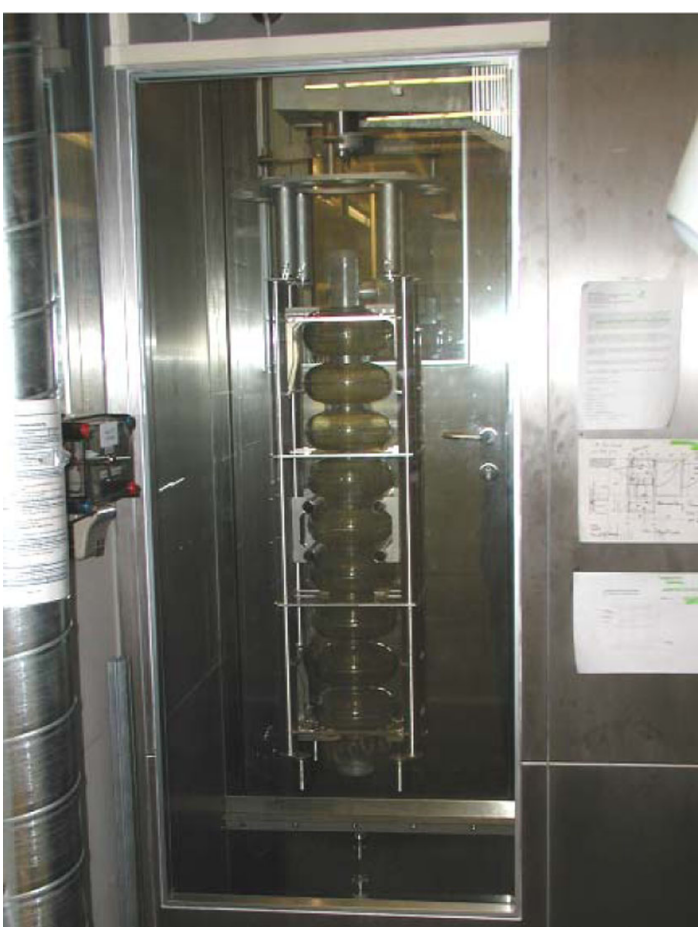

FIG. 24. (Color) View on the new HPR cabinet.

\section{HPR pump}

For pressurizing the UP water a commercial turbine type Sunflo P $3000^{2}$ (Fig. 25) is installed. The turbine with a rotation speed of 19.000 turns per minute shows maximum pressure variations of $\leq 1$ bar at 100 bar system pressure and a capacity of up to $6 \mathrm{~m}^{3}$ /hour at 100 bar. This design allows supply of up to five HPR stands with a DESY standard nozzle head installed.

Gearbox and turbine are separated by a control space. The turbine and turbine body are electropolished and lubrication free. The turbine is equipped with whole metal bearing, lubricated, and controlled by permanent water flush. Drain water from the turbine as well as abrasives from the bearing are drained by a dedicated flush port. This port is usable for quality control of the bearing and leakage of the gearbox seals.

The water pressure can be regulated from 30 to 100 bar (maximum) by a frequency driver with a setting precision of 1 bar.

\section{Motion unit: General layout}

The existing HPR system, in use at DESY since 1993, is based on the motion of a cane actuated by a motion unit installed underneath. This design bears the risk that the cane is contaminated by particulates and abrasives from seals of the drain system and can be guided into the cavity interior.

\footnotetext{
${ }^{2}$ Sunflo P 3000 is a product of SPX Process Equipment by Sundyne Corporation. The German distributor is Bran + Luebbe GmbH, Werkstrasse 4, D-22844 Norderstedt, Germany.
}

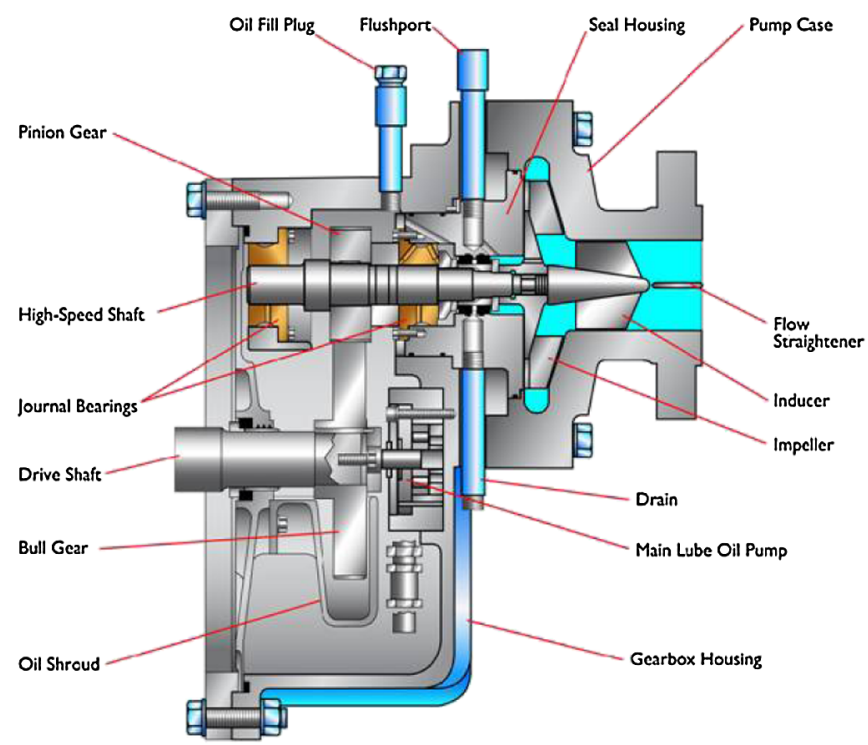

Sunflo P3000 Pump

FIG. 25. (Color) Cross section of the HPR pump.

The new HPR design fixes the position of the spraying cane while the cavity performs the three-dimensional motion. A central post, housed in a separate part of the cabinet, takes all forces coming from motors, motion systems, and the load of a cavity. The cabinet of the central post is ventilated by the exhausting air of the HPR cabinet. The central post deformation under the full load capacity of $150 \mathrm{~kg}$ is measured to be less than $0.2 \mathrm{~mm}$ in respect to the parallel resonator axis. For vertical motion, a stepping motor with gearbox and spindle drive is installed. The rotation of the resonator, hanging on the central bearing ${ }^{3}$ (Fig. 26), is realized by a timing belt drive made from Teflon ${ }^{\circledR}$. The timing belt is driven by a stepping motor connected to the central post.

To prevent abrasives originating from the timing belt or ball bearing from entering the ISO 4 area, the rotating parts are sealed by rotational shaft seals made from polytetrafluorethylen.

\section{E. Ball bearing of the central cavity fixture}

The central part of the cavity fixture, a lever arm motion unit, is holding the resonator during rinsing. The lever arm is located on top of the cavity. During the design phase, a 1:1 plastic model was fabricated to study the influence of the fixture to the laminar flow. A ball bearing made of ceramic balls and stainless bearing housing (Fig. 27) is installed in the center of the lever arm. The cavity adapter, as well as the rotational driving unit, is connected to this central ball bearing.

\footnotetext{
${ }^{3}$ The ball bearing is a product by $\mathrm{E} \& \mathrm{~K}$ Wälzlager $\mathrm{GmbH}$, Max-Plank Strasse, D-52499 Baesweiler, Germany.
} 


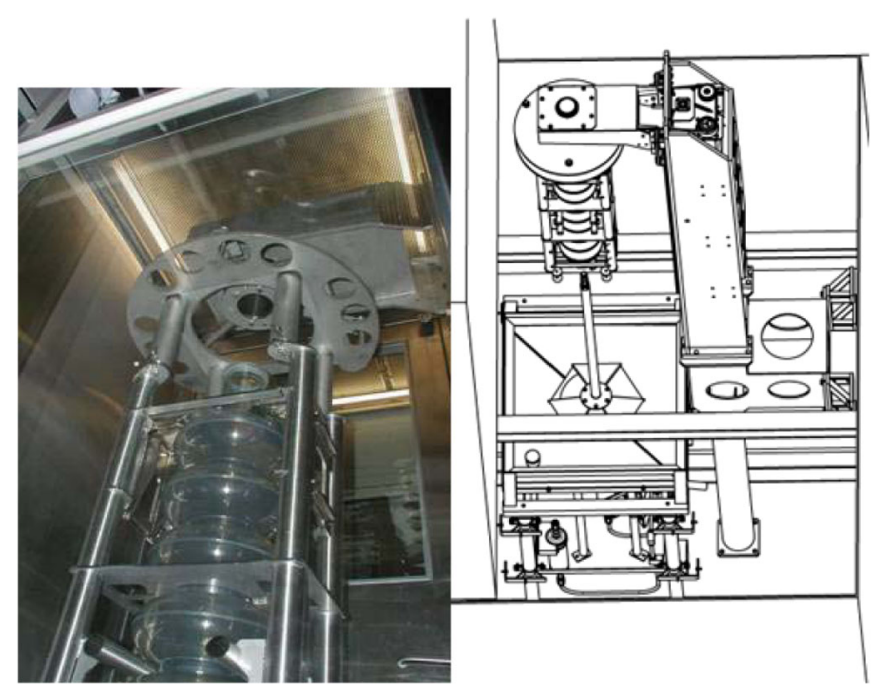

FIG. 26. (Color) Left: View on the cavity adapter lever arm. Right: Schematic of the HPR system with lever arm motion unit and cavity installed.

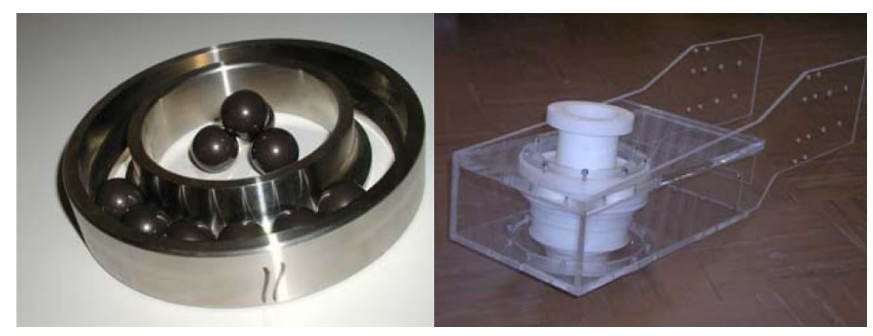

FIG. 27. (Color) Left: View on the ceramic central ball bearing. Right: 1/1 plastic model of the cavity adapter lever arm.

\section{F. UP water supply}

The new HPR pump is capable of feeding up to five HPR stands in parallel. A minimum water flow of $6 \mathrm{~m}^{3} / \mathrm{h}$ is required by the turbine in order to prevent cavitations, even if the current setup at DESY only consumes 1 to $1.5 \mathrm{~m}^{3} / \mathrm{h}$. The DESY UP water plant allows feeding up to a maximum of $20 \mathrm{l} / \mathrm{min}$ of UPW. To suppress strong flow variations in the existing UPW plant at startup of the new HPR a separate UPW supply is installed. It is fed continuously from the main UPW plant. The HPR UPW system buffers the turbine water supply in a tank of 5001 storage volume, leveling the nonlinear water consumption during startup and shutdown of the turbine. In addition, the tank allows continuous circulation of the UPW through all piping and valves of the HPR system during stand-by operation. The water quality is controlled by on-line total organic carbon, resistivity, and temperature control sensors.

\section{G. Computer control and visualization}

The HPR stand is controlled and steered by a Siemens S7 PLC (Siemens Industry Automation and Drive

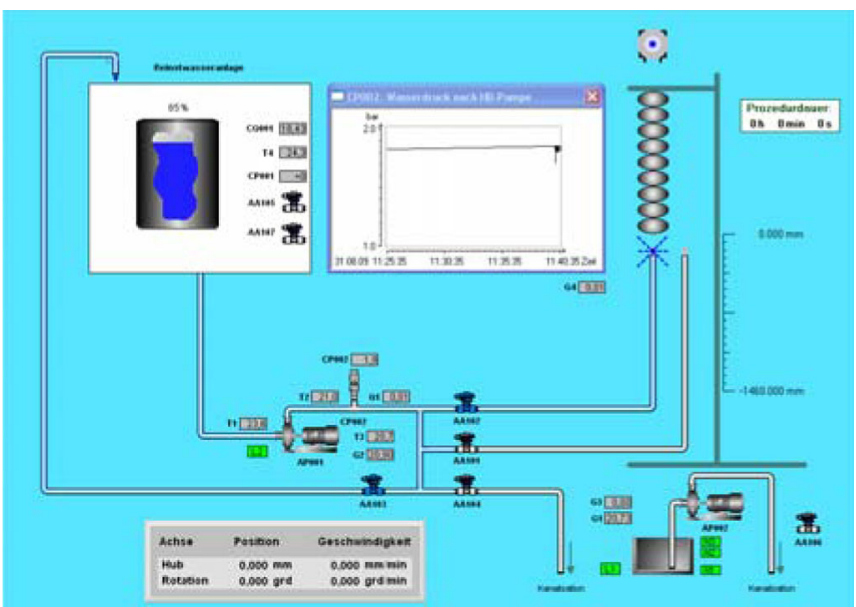

FIG. 28. (Color) View on the control and steering panel.

Technologies). For communication, display of parameters and manual operation the PLC can be remotely controlled by a PC. All system parameters as well as the current position of the cavity (rotation and vertical position) are displayed (Fig. 28) and stored on disk.

\section{H. Operation experience and cavity performance}

The new HPR system was commissioned successfully. More than 300 HPR cycles of $2 \mathrm{~h}$ duration have been performed, and the system has proven its robustness and reproducibility.

In HP rinse treatments redone after rf measurements of cavities with the new HPR stand, improvements on field emission limits were observed (Fig. 29). Since 2008 the statistics of cavity treatments exclusively performed with the new HPR system show an average maximum acceleration gradient of $31.8 \mathrm{MV} / \mathrm{m}$ with gradients up to $41 \mathrm{MV} / \mathrm{m}$. The average acceptance gradient is $27.5 \mathrm{MV} / \mathrm{m}$ with respect to the allowed radiation $\left(<10^{-2} \mathrm{mGy} / \mathrm{min}\right)$ for FLASH (Fig. 30).

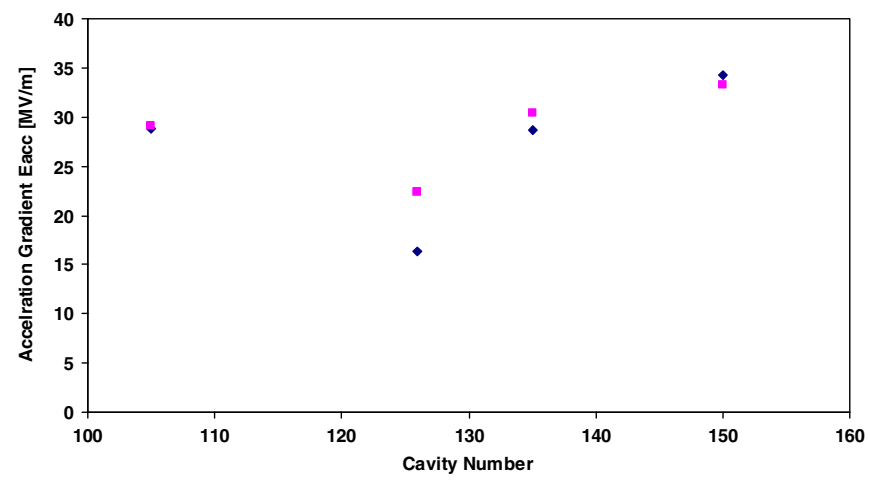

FIG. 29. (Color) The $\mathrm{rf}$ test results of nine-cell cavities retreated with the new HPR system: red = Eacc after retreatment; blue = Eacc before treatment. 


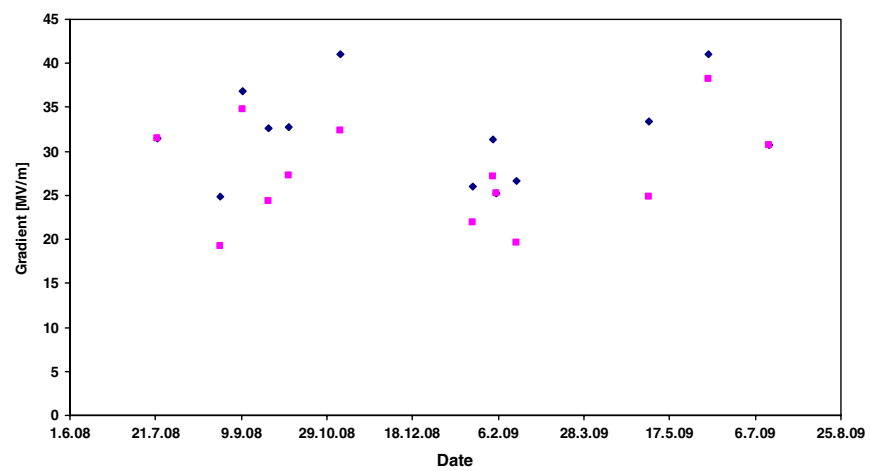

FIG. 30. (Color) The rf test results of nine-cell cavities exclusively HP rinsed in the new HPR system: blue $=$ maximum acceleration gradient; red $=$ acceptance gradient EACpt for FLASH $0.01 \mathrm{mGy} / \mathrm{min}$.

\section{SUMMARY}

The European XFEL is based on a superconducting linac comprising 800 accelerator cavities. Test results of cavities undergoing the main EP in industrial company facilities show no differences with respect to cavities treated in the EP DESY facilities. The process of industrial main EP is reproducible. For an optimized cavity preparation, the helium tank is installed at an early state of preparation to the cavity. The vertical rf test as well as the $120^{\circ} \mathrm{C}$ baking is done on cavities dressed with a helium tank and ready for module installation. The rf test results show that the proposed cavity preparation results in the same statistical distribution of cavity performance as previously found. It is shown that the average gradients of cavities undergoing surface preparation with either final EP or BCP flash can be used for the XFEL project.

All conceptual requirements during development, design, programming, commissioning, and conformity assessment of a new rf-measurement machine could be fulfilled. The very successful use of HAZEMEMA during cavity fabrication for FLASH at industry proved the expected significant reduction of necessary working time compared to the former manual device. It could be shown that operation by a nonexpert is possible. Two more machines are under fabrication and will be used for the series fabrication of 800 cavities for the European XFEL.

A series of four new efficient tuning machines was successfully developed in a collaboration among FNAL, KEK, and DESY. The testing and commissioning of these machines is in progress. With the cavity series production start of 800 cavities for the European XFEL, these machines will have to demonstrate their capabilities.

A new high pressure rinsing system was developed and is operational. It is shown that the general design of central ceramic ball, motion units, lever arm, and cavity adapter are applicable in class ISO 4 conditions. A turbine in use as an HPR pump leads to a vibration-free feeding of the spraying cane. In more than 300 HPR cycles of 2 hours length, the new HPR system showed a robust and reproducible behavior. Maximum accelerating gradients of up to $41 \mathrm{MV} / \mathrm{m}$ have been reached. The new HPR system is qualified and became part of the ongoing cavity preparation at DESY.

\section{ACKNOWLEDGMENTS}

The XFEL cavity fabrication, preparation, and testing is a collaborative effort. Thus the authors would like to thank the complete team consisting of experts from different institutes. We acknowledge the technical support as well as the good cooperation of: Company Damker (Hamburg, Germany), Company ZSI Zertz + Scheid (Gummersbach, Germany), and Company CE-CON (Bremen, Germany). We also appreciate the support of Research Instruments $\mathrm{GmbH}$ (former ACCEL Instruments $\mathrm{GmbH}$ ) and Zanon SPA during the rf measurements in the cavity production. We give special thanks to K. Lando for proofreading this paper.

[1] DESY XFEL Project Group and European XFEL Project Team, DESY 2006-97, Hamburg, 2007.

[2] H. Weise, in Proceedings of the 14th Workshop on RF Superconductivity, Berlin, Germany, 2009 (Helmholtz Zentrum für Materialien und Energie, Berlin, 2009), paper MOOAAU02.

[3] B. van der Horst et al., in Proceedings of the 13th Workshop on RF Superconductivity, Beijing, China, 2007, edited by Jiankui Hao, Senlin Huang, and Kui Zhao (Peking University, Beijing, 2007), paper TUP30.

[4] B. van der Horst et al., in Proceedings of the 14th Workshop on RF Superconductivity, Berlin, Germany, 2009, Ref. [2], paper THPPO072.

[5] D. Reschke et al., in Proceedings of the 14th Workshop on RF Superconductivity, Berlin, Germany, 2009, Ref. [2], paper TUPPO051.

[6] J. Iversen et al., in Proceedings of the 14th Workshop on RF Superconductivity, Berlin, Germany, 2009, Ref. [2], paper THPPO071.

[7] J.H. Thie et al., in Proceedings of the 14th Workshop on RF Superconductivity, Berlin, Germany, 2009, Ref. [2], paper THPPO074.

[8] A. Matheisen et al., in Proceedings of the 14th Workshop on RF Superconductivity, Berlin, Germany, 2009, Ref. [2], paper THPPO073.

[9] A. Schmidt et al., in Proceedings of the 14th Workshop on RF Superconductivity, Berlin, Germany, 2009, Ref. [2], paper THPPO040.

[10] A. Matheisen et al., in Proceedings of the 12th Workshop on RF Superconductivity, Ithaca, NY, 2005, edited by S. Belomestnykh, M. Liepe, and H. Padamsee, paper ThP08.

[11] M. Schmoekel et al., in Proceedings of the 13th Workshop on RF Superconductivity, Beijing, China, 2007, Ref. [3], paper TUP31.

[12] S. Aderhold, in Proceedings of the 14th Workshop on RF Superconductivity, Berlin, Germany, 2009, Ref. [2], paper TUPPO035. 
[13] W. D. Möller and J. Sekutowicz (private communication).

[14] G. Kreps, D. Proch, and J. Sekutowicz, in Proceedings of the 9th Workshop on RF Superconductivity, Santa Fe, USA, 1999 (Los Alamos National Laboratory, Los Alamos, 1999), paper WEP031.
[15] J. Sekutowicz et al., in Proceedings of the 4th Workshop on RF Superconductivity, Tsukuba, Japan, 1989 (National Laboratory for High Energy Physics, Tsukuba, 1990), p. 849. 